\title{
Making a Positive Diagnosis of Intestinal Tuberculosis with the Aid of New Biologic and Histologic Features: How Far Have We Reached?
}

\author{
Vatsal Mehta Devendra Desai Philip Abraham Camilla Rodrigues \\ Divisions of Medical Gastroenterology and Microbiology, P.D. Hinduja Hospital, Mumbai, India
}

\section{Keywords}

Intestinal tuberculosis · Mycobacteria Growth Indicator

Tube culture $\cdot$ Colonoscopy

\begin{abstract}
Background: The diagnosis of intestinal tuberculosis (TB) and its differentiation from Crohn's disease (CD) remain a challenge. We review here in detail the various methods for the diagnosis of intestinal TB. Summary: Colonoscopy findings in intestinal TB are useful and suggestive; histopathology of colonoscopic biopsies is contributory but rarely confirmatory. Increasing the number of colonoscopic biopsies increases the histological yield. Recent culture methods that have improved the yield for TB offer hope. Mycobacteria Growth Indicator Tube (MGIT) culture is now the standard of care as its yield is superior to that of the traditional Lowenstein-Jensen medium. Increasing the number of colonoscopic biopsy samples for MGIT culture can increase the yield. The culture and histology are complimentary. Even then a significant proportion of patients do not have a positive diagnosis of intestinal TB. Scoring systems have been developed with a sensitivity and specificity of 90 and $60 \%$, respectively, but their utility in routine practice is yet to be established. Similarly, the ratio of visceral fat to total fat is helpful in differentiating CD from intestinal TB. Polymerase
\end{abstract}

chain reaction has been used but its value seems uncertain. Gene Xpert ${ }^{\circledR}$ in an emerging technique that has been found to be useful in the diagnosis of pulmonary TB, and its utility in intestinal TB needs to be looked at. Newer technologies like TB-LAMP (loop-mediated isothermal amplification) need to be assessed in clinical studies. Key Message: Optimization of the present diagnostic tools (taking an adequate number of biopsies for histology and culture) and study of newer techniques to learn their actual utility seems to be the way forward.

(c) 2019 S. Karger AG, Basel

\section{Introduction}

Worldwide, approximately 9.6 million people were estimated to have been afflicted with tuberculosis (TB) in 2014 , including 5.4 million men, 3.2 million women, and 1.0 million children [1]. Geographically, the burden of TB is highest in Asia and Africa. India and China together account for almost $40 \%$ of the world's cases. In these 2 countries, less than 1 in 10 cases has multidrug-resistant $\mathrm{TB}$, but a scale-up is expected in the next 3 years [2]. The proportion of patients with extrapulmonary TB is $8-13 \%$, and among these abdominal $\mathrm{TB}$ accounts for $7 \%$ of the patients [3].

\section{KARGER}

(c) 2019 S. Karger AG, Basel

E-Mail karger@karger.com

www.karger.com/iid
Dr. Devendra Desai

P.D. Hinduja Hospital

Veer Savarkar Marg, Mahim

Mumbai 400016 (India)

E-Mail devendracdesai@gmail.com 
Table 1. Histology findings in intestinal TB

\begin{tabular}{lllll}
\hline Study & $\begin{array}{l}\text { Patients } \\
\text { with TB, } n\end{array}$ & $\begin{array}{l}\text { Granuloma, } \\
\%\end{array}$ & $\begin{array}{l}\text { Caseation, } \\
\%\end{array}$ & $\begin{array}{l}\text { Nonspecific } \\
\text { features, } \%\end{array}$ \\
\hline Vij et al. [9] & 37 & 67 & 13 & 33 \\
Shah et al. [10] & 50 & 74 & 18 & 20 \\
Pulimood et al. [11] & 61 & 46 & 18 & 22 \\
Kirsch et al. [12] & 18 & 78 & 22 & 38 \\
Amarapurkar et al. [13] & 26 & 57 & 34 & \\
\hline
\end{tabular}

The numbers are not mutually exclusive.

It is generally believed that the incidence of Crohn's disease $(C D)$ is increasing in countries afflicted by TB [4], and differentiating intestinal TB from $\mathrm{CD}$ is often a challenge [5]. Isolation of acid-fast bacilli (AFB) is a vital step in the diagnosis of intestinal $\mathrm{TB}$ and its differentiation from $\mathrm{CD}$; a positive culture also enables drug sensitivity testing and thus the diagnosis of multidrug-resistant TB.

Digestive tract sarcoidosis can very rarely mimic TB or $\mathrm{CD}$. The most common site of involvement is the stomach, followed by the colon. Patients present with abdominal pain, weight loss, nausea/vomiting, diarrhea, and digestive bleeding. Differentiating digestive tract sarcoidosis from CD and intestinal TB can be difficult. Thoracic adenopathy, other systemic features, and a negative TB culture may lead to suspicion of this relatively rare disease. However, histological examination of large specimens may yield the final diagnosis [6-8].

\section{Histological Diagnosis of Intestinal TB}

The availability of colonoscopic biopsy has significantly reduced the need for surgical/laparoscopic access for tissue. Histology is often not definitive for a diagnosis of $\mathrm{TB}$, and culture remains the gold standard.

Histological features of TB include granulomas with caseating necrosis, conglomerate epithelioid histiocytes, and disproportionate submucosal inflammation. As shown in Table 1, the classical histological features are seen in only $13-33 \%$ of patients; the presence of granulomas in a clinically suggestive setting helps to reach the diagnosis in $57-74 \%$ of the patients [9-13].

A larger number of biopsy samples available for histology is naturally expected to increase the diagnostic yield for TB; however, this comes at the cost of more time for biopsies and processing. Various authors have recommended 4-10 biopsies. Yönal and Hamzaoğlu [14], in a review, recommended at least 8 colonoscopic biopsies for a satisfactory histological evaluation, based on data given in various studies.

\section{AFB Culture in Intestinal TB}

There are 3 types of AFB culture medium, i.e., eggbased (the traditional Lowenstein-Jensen [LJ] medium), agar-based (e.g., Middlebrook 7H10 or 7H11), and liquid (Middlebrook 7H12 and other commercially available broths) media.

Newer methods include the radiometric BACTEC 460 system, the MGIT BACTEC 960 system, and the EPS II system. The BACTEC system, developed by Becton Dickinson (New York, NY, USA), is based on generation of radioactive carbon dioxide from substrate palmitic acid [15]. The Mycobacteria Growth Indicator Tube (MGIT) system (also developed by Becton Dickinson) is based on a nonradioactive method using fluorochromes for detection of growth and drug screening. This system helps in early detection (7-12 days) of mycobacterial growth and has been reported to be useful for drug susceptibility testing [16].

The MGIT system has several benefits besides being radiation free. Full automation eliminates loading and unloading of tubes and thus minimizes the risk of bottle breakage; $\mathrm{CO}_{2}$ tanks are not required; the noninvasive monitoring of cultures eliminates the possibility of crosscontamination; the use of screw caps on the tubes eliminates the need for use of needles and thus the risk of inadvertent needle pricks [17-19]. Hence, the MGIT system is today the preferred method of isolation of the TB organism.

The yield of AFB culture on LJ medium is poor, ranging from 6 to 48\% [9, 10]. Morgan et al. [20] and Bhargava et al. [21] compared the bactenecin (BACTEC) system with Middlebrook 7H100 and LJ media (Table 2). 
Table 2. Comparison of traditional culture methods and the MGIT BACTEC 960 system

\begin{tabular}{|c|c|c|c|c|c|c|c|}
\hline \multirow[t]{2}{*}{ Study } & \multirow{2}{*}{$\begin{array}{l}\text { Ratio of positive } \\
\text { samples/ } \\
\text { total samples }\end{array}$} & \multicolumn{2}{|l|}{ LJ medium } & \multicolumn{2}{|c|}{ Middlebrook 7H10 } & \multicolumn{2}{|c|}{ MGIT BACTEC 960} \\
\hline & & $\begin{array}{l}\text { positive } \\
\text { samples, \% }\end{array}$ & $\begin{array}{l}\text { recovery } \\
\text { time, } \\
\text { days }\end{array}$ & $\begin{array}{l}\text { positive } \\
\text { samples, \% }\end{array}$ & $\begin{array}{l}\text { recovery } \\
\text { time, } \\
\text { days }\end{array}$ & $\begin{array}{l}\text { positive } \\
\text { samples, \% }\end{array}$ & $\begin{array}{l}\text { recovery } \\
\text { time, } \\
\text { days }\end{array}$ \\
\hline Morgan et al. $[20]^{\mathrm{a}}$ & $71 / 2,165$ & 44 & 26 & 39 & 26 & 51 & 13 \\
\hline Bhargava et al. [21] & $58 / 100$ & 53 & 35 & 39 & 42 & 53 & 20 \\
\hline
\end{tabular}

${ }^{\mathrm{a}}$ Included all smear-negative pulmonary and extrapulmonary samples excluding CSF and urine. ${ }^{\mathrm{b}}$ Included 73 pulmonary and 27 extrapulmonary samples.

Table 3. AFB culture positivity on LJ medium and the BACTEC method in intestinal TB

\begin{tabular}{lllcl}
\hline Study & $\begin{array}{l}\text { Sample } \\
\text { size, } n\end{array}$ & Source & Culture positivity, \% & Method \\
\hline Shah et al. [10] & 50 & Colonoscopy & 6 & LJ medium \\
Kirsch et al. [12] & 18 & Colonoscopy & 11 & BACTEC \\
Shah et al. [22] & 28 & Colonoscopy + surgery & 48 & LJ medium \\
Samant et al. [23] & 62 & Colonoscopy & 50.8 & BACTEC \\
\hline
\end{tabular}

They reported the highest AFB culture yield and the fastest growth on the MGIT 960 system.

Studies based on surgical specimens have shown higher TB culture rates. Shah et al. [22] studied 9 patients with surgical biopsies and 18 with colonoscopic biopsies. The BACTEC method was superior, with $76 \%$ positivity as compared to $48 \%$ on LJ medium. In this study surgical specimens increased the AFB culture positivity.

\section{Number of Biopsies and Increasing Yield}

Table 3 shows the AFB culture positivity in patients with intestinal TB. Earlier studies had a much lower culture positivity rate [22]. There has been a recent gradual improvement in the yield from colonoscopic biopsies to nearly $51 \%$ [23]. As mentioned earlier, the yield of histopathology can be increased by taking 8 biopsies. Similarly, 8 biopsies can increase the yield of AFB culture as compared to 4 biopsies. In an earlier study, we examined 190 patients suspected to have intestinal TB, over a period of 2 years, and 70 patients had confirmed TB based on histology and/or AFB culture. The study revealed that taking 8 biopsies increased the diagnostic yield by $11.4 \%$ as compared to 4 biopsies [24].

\section{Combining Histology and AFB Culture for Diagnosis of Intestinal TB}

Given the limitations of histology and AFB culture, combining the two methods may improve the diagnostic yield. This will reduce the number of patients who receive empiric anti-TB treatment. The combined yield has improved from $75 \%$ in earlier studies to $92 \%$ in a recent study (Table 4, footnote d) $[9,10,13,22,23,25]$.

\section{Scoring for Differentiation between Intestinal TB and $C D$}

Makharia et al. [26] devised a score on the basis of regression coefficients of the final multivariate logistic model, which varied from 0.3 to 9.3. Higher scores predicted a greater likelihood of intestinal TB. With the cutoff at 5.1, the area under ROC in the validation data set was $89.2 \%$ (95\% CI $0.79-0.99)$ and the sensitivity and specificity were $90 \%$ (95\% CI 66.9-98.2) and 60\% (95\% CI 36.4-80.0), respectively. The low specificity restricts the positive diagnosis. 
Table 4. Diagnostic yield of histology and AFB culture in intestinal TB

\begin{tabular}{|c|c|c|c|c|c|}
\hline Study & $\begin{array}{l}\text { Patients with } \\
\text { ileocolonic TB, } n\end{array}$ & $\begin{array}{l}\text { Histology, } \\
n(\%)\end{array}$ & $\begin{array}{l}\text { AFB culture } \\
\text { positivity, } n(\%)\end{array}$ & $\begin{array}{l}\text { Culture } \\
\text { method }\end{array}$ & $\begin{array}{l}\text { Combined } \\
\text { diagnostic yield, \% }\end{array}$ \\
\hline Vij et al. [9] & 28 & $21(75)$ & $13(46)$ & LJ medium & $75^{\mathrm{a}}$ \\
\hline Shah et al. [10] & 50 & $40(80)$ & $3(6)$ & LJ medium & 80 \\
\hline Leung et al. [25] & 23 & $3(13)$ & $17(73)$ & BACTEC & 82 \\
\hline \multirow[t]{2}{*}{ Shah et al. $[22]^{\mathrm{c}}$} & 28 & NA & 48 & LJ medium & NA \\
\hline & & & 76 & BACTEC & \\
\hline
\end{tabular}

NA, not available. ${ }^{a}$ Included all gastrointestinal TB cases. ${ }^{b}$ Included parameters like clinical score and TB PCR. ${ }^{c}$ Included only histology-positive cases. ${ }^{\mathrm{d}}$ In these studies the gold standards for the diagnosis of intestinal TB were: culture positivity and, in presence of convincing clinical features, imaging evidence and histological features with a positive response to treatment documented by repeat imaging or colonoscopy.

Table 5. Sensitivity and specificity of TB PCR in intestinal TB

\begin{tabular}{llrr}
\hline Study & Type of sample & Sensitivity, \% & Specificity, \% \\
\hline Amarapurkar et al. [13] & Paraffin-embedded biopsy specimens & 21.6 & 95 \\
Hillemann et al. [29] & Paraffin-embedded biopsy specimens & 66 & 100 \\
Pulimood et al. [30] & Deparaffinized biopsy specimens (in situ PCR) & 30 & 95 \\
Gan et al. [31] & Paraffin-embedded biopsy specimens & 64.1 & 100 \\
\hline
\end{tabular}

\section{Visceral Fat/Subcutaneous Fat Ratio on CT Scan}

Two recent retrospective studies have examined the ratio of visceral fat to subcutaneous fat in patients with CD and intestinal TB. Similarly, the ratio of visceral fat to total fat has also been studied. The ratio of visceral fat to subcutaneous fat and the ratio of visceral fat to total fat are higher in $\mathrm{CD}$ as compared to intestinal TB. For a VF/ TF cut-off value of 0.46 , the sensitivity and specificity for the diagnosis of CD were 42.1 and $93.3 \%$ respectively, with positive and negative predictive values of 88.9 and $56.0 \%$, respectively [27]. A cut-off of 0.63 for the VF/SC ratio had a high sensitivity of $82 \%$ and a specificity of $81 \%$ in differentiating $\mathrm{CD}$ and intestinal TB [28].

\section{Newer Diagnostic Modalities}

\section{TB Polymerase Chain Reaction Methods}

Various polymerase chain reaction (PCR) methods have been developed for the detection of specific sequenc- es of Mycobacterium tuberculosis and other mycobacteria. These assays may target DNA or rRNA. Three main types of PCR techniques are available, i.e., DNA targeting probe, $r R N A$ targeting probe, and gene amplification assay [28]. Although the specificity of PCR assay is very high (Table 5), its low sensitivity has limited its use in clinical practice $[13,29,30,31]$. The small quantity of tissue available in mucosal biopsy specimens and the limited number of sections used for DNA extraction may limit the copy numbers of $M$. tuberculosis DNA; hence the low sensitivity.

\section{Gene Xpert ${ }^{\circledR}$ Assay}

The Xpert MTB/RIF (rifampicin) assay is a fully automated real-time PCR-based test designed for rapid and simultaneous detection of $M$. tuberculosis and mutations associated with rifampicin resistance, and the result is available within $2 \mathrm{~h}[32,33]$. The test is expensive but it is marketed as a point-of-care test and requires virtually no training or laboratory infrastructure. Pimkina et al. [34] conducted a study on sputum and bronchoalveolar lavage specimens and showed that the sensitivity of the Gene 
Xpert assay is nearly $100 \%$ when AFB are detected on a smear. Smear-negative but culture-positive specimens had a sensitivity of $85 \%$. For smear- and culture-negative specimens, the sensitivity of the assay was only $8 \%$. Thus, the advantage of the Gene Xpert assay is achievement of a rapid diagnosis at a point of care and additional information about drug resistance, rather than an additional yield. An Indian study [35] included pulmonary $(n=384)$ and extrapulmonary $(n=761)$ samples. Among the latter, the sensitivity of the Gene Xpert assay was $88 \%$ and its specificity was $91 \%$. Kumar et al. [36] found that the sensitivity, the specificity, the positive predictive value, and the negative predictive value of the Gene Xpert assay were 8.1, 100, 100 , and $64.2 \%$, respectively, in intestinal TB cases.

\section{Loop-Mediated Isothermal Amplification Test for TB}

This method is fast, results can be detected by the naked eye, and it does not require expensive equipment. Current TB-LAMP (loop-mediated isothermal amplification) assays are based on amplification of MTBC genomic DNA targeting the gyrB and IS6110 genes. A study done by Kumar et al. [37] on 118 clinical samples (41 pulmonary samples [sputum, $n=29$; bronchoalveolar lavage, $n=7$; and gastric aspirate, $n=5$ ] and 77 extrapulmonary samples [CSF, $n=28$; pus, $n=11$; pleural fluid, $n=15$; ascitic fluid, $n=2$; lymph node aspirate, $n=7$; urine, $n=$ 5 ; abscess pus, $n=3$; and other body fluids, $n=6]$ ). LAMP showed a higher detection rate $(52.5 \%)$ as compared to mPCR (multiplex PCR) (44\%) and culture (30.5\%). On culture-positive and mPCR-positive samples, the sensitivity of LAMP was $100 \%$ and its specificity was $96.1 \%$. Similarly, Bojang et al. [38] reported sensitivities and specificities of 98.6 and 99\% for TB-LAMP, 91.1 and $100 \%$ for MGIT culture, and 99.1 and $96 \%$ for Gene Xpert for the diagnosis of TB. These new studies have not significantly improved on sensitivity or specificity for the diagnosis of TB. However, these studies are useful for early diagnosis of $M$. tuberculosis and identification of drugresistant strains.

\section{Proteomic Profiling}

The differently expressed protein peaks analyzed by serum proteome with weak cationic magnetic beads combined with the matrix-assisted laser desorption/ionization-time of flight mass spectrometry (MALDI-TOFMS) technique can effectively distinguish $\mathrm{CD}$ patients from healthy controls (HC), intestinal TB patients from $\mathrm{HC}$, and $\mathrm{CD}$ patients from Intestinal TB patients. Zhang et al. [39] conducted a study on $30 \mathrm{CD}$ patients, 21 intestinal $\mathrm{TB}$ patients, and $30 \mathrm{HC}$ and concluded that:
- the diagnostic model between CD patients and HC consisting of 4 protein peaks (M/Z 4964, 3029, 2833, and 2900) had a sensitivity and specificity of $96 \%$;

- the diagnostic model between intestinal TB patients and HC comprising 4 protein peaks (M/Z 3030, 2105, 2545 , and 4210) had a sensitivity and specificity of 93 and $95 \%$, respectively; and

- the differential diagnostic model between CD patients and intestinal TB patients comprising 3 protein peaks (M/Z 4267, 4223, 1541 and) had a sensitivity and specificity of 76 and $80 \%$, respectively [39].

Similarly, protein expression in macroscopically affected mucosa is different in CD and Intestinal TB. Rukmangadachar et al. [40] identified 63 proteins differentially expressed in colonic mucosa of patients with $\mathrm{CD}$ and intestinal TB and 6 proteins used for validation employing immunohistochemistry in a larger cohort of patients. However, multiple large-scale studies will be required for further validation of these findings [40].

In summary, a positive diagnosis of intestinal $\mathrm{TB}$ remains a challenge due to limitations in histology and culture, which are the presently relied-upon methods for the diagnosis of intestinal TB. With an increasing prevalence of $\mathrm{CD}$ in countries like India, differentiating this disease from TB is increasingly important. The refinement in culture has improved the yield of culture to about $50 \%$. The combination of histology and culture is complimentary. Optimization of the present diagnostic tools (taking an adequate number of biopsies for histology and culture) and study of newer techniques to learn their actual utility seems to be the way forward.

\section{Statement of Ethics}

Since this is a review, it has not involved any patients/animals or any intervention. This article has not been subjected to institutional review board review.

\section{Disclosure Statement}

None of the authors have any conflict of interest. 


\section{References}

1 Executive Summary. Global tuberculosis report. 2015.

2 World Health Organization. Global tuberculosis report 2012. Geneva: WHO; 2012. p. 258

3 Wares F, Balasubramanian R, Mohan A. Extrapulmonary tuberculosis: management and control. In: Agarwal SP, Chauhan LS, editors. Tuberculosis control in India. New Delhi: Elsevier; 2005. p. 95-114.

4 Sharma MP, Bhatia V. Abdominal tuberculosis. Indian J Med Res. 2004 Oct;120(4):30515.

5 Economou M, Zambeli E, Michopoulos S. Incidence and prevalence of Crohn's disease and its etiological influences. Ann Gastroenterol. 2009;22:158-67.

6 Sorrentino D, Avellini C, Zearo E. Colonic sarcoidosis, infliximab, and tuberculosis: a cautionary tale. Inflamm Bowel Dis. $2004 \mathrm{Jul}$ 10(4):438-40.

7 Ghrenassia E, Mekinian A, Chapelon-Albric C, Levy P, Cosnes J, Sève P, et al.; Groupe Sarcoïdose Francophone. Digestive-tract sarcoidosis: french nationwide case-control study of 25 cases. Medicine (Baltimore). 2016 Jul;95(29):e4279.

8 Erra P, Crusco S, Nugnes L, Pollio AM, Di Pilla G, Biondi G, et al. Colonic sarcoidosis: unusual onset of a systemic disease. World J Gastroenterol. 2015 Mar;21(11):3380-7.

9 Vij JC, Malhotra V, Choudhary V, Jain NK, Prasad G, Choudhary A, et al. A clinicopathological study of abdominal tuberculosis. Indian J Tuberc. 1992;39:213-20.

10 Shah S, Thomas V, Mathan M, Chacko A, Chandy G, Ramakrishna BS, et al. Colonoscopic study of 50 patients with colonic tuberculosis. Gut. 1992 Mar;33(3):347-51.

11 Pulimood AB, Ramakrishna BS, Kurian G, Peter S, Patra S, Mathan VI, et al. Endoscopic mucosal biopsies are useful in distinguishing granulomatous colitis due to Crohn's disease from tuberculosis. Gut. 1999 Oct;45(4):537-41.

12 Kirsch R, Pentecost M, Hall PM, Epstein DP, Watermeyer G, Friederich PW. Role of colonoscopic biopsy in distinguishing between Crohn's disease and intestinal tuberculosis. J Clin Pathol. 2006 Aug;59(8):840-4.

13 Amarapurkar DN, Patel ND, Rane PS. Diagnosis of Crohn's disease in India where tuberculosis is widely prevalent. World J Gastroenterol. 2008 Feb;14(5):741-6.

14 Yönal O, Hamzaoğlu HÖ. What is the most accurate method for the diagnosis of intestinal tuberculosis? Turk J Gastroenterol. 2010 Mar;21(1):91-6.

15 Venkataraman P, Herbert D, Paramasivan $\mathrm{CN}$. Evaluation of the BACTEC radiometric method in the early diagnosis of tuberculosis. Indian J Med Res. 1998 Oct;108:120-7.

16 Tortoli E, Cichero P, Piersimoni C, Simonetti MT, Gesu G, Nista D. Use of BACTEC MGIT 960 for recovery of mycobacteria from clinical specimens: multicenter study. J Clin Microbiol. 1999 Nov;37(11):3578-82.
17 Woods GL, Fish G, Plaunt M, Murphy T. Clinical evaluation of difco ESP culture system II for growth and detection of mycobacteria. J Clin Microbiol. 1997 Jan;35(1):121-4.

18 Rodrigues C, Shenai S, Sadani M, Sukhadia N, Jani M, Ajbani K, et al. Evaluation of the bactec MGIT 960 TB system for recovery and identification of Mycobacterium tuberculosis complex in a high through put tertiary care centre. Indian J Med Microbiol. 2009 Jul-Sep; 27(3):217-21.

19 Bemer P, Palicova F, Rüsch-Gerdes S, Drugeon $\mathrm{HB}$, Pfyffer GE. Multicenter evaluation of fully automated BACTEC Mycobacteria Growth Indicator Tube 960 system for susceptibility testing of Mycobacterium tuberculosis. J Clin Microbiol. 2002 Jan;40(1):150-4.

20 Morgan MA, Horstmeier CD, DeYoung DR, Roberts GD. Comparison of a radiometric method (BACTEC) and conventional culture media for recovery of mycobacteria from smear-negative specimens. J Clin Microbiol. 1983 Aug;18(2):384-8.

21 Bhargava AJ, Jain A, Agarwak SK. A comparison of liquid and solid culture media with radiometric system for detection of mycobacteria in clinical specimens. Indian J Tuberc. 2001;48:9-12.

22 Shah SR, Shenai S, Desai DC, Joshi A, Abraham $\mathrm{P}$, Rodrigues C. Comparison of Mycobacterium tuberculosis culture using liquid culture medium and Lowenstein Jensen medium in abdominal tuberculosis. Indian J Gastroenterol. 2010 Nov;29(6):237-9.

23 Samant H, Desai D, Abraham P, Joshi A, Gupta T, Rodrigues C, et al. Acid-fast bacilli culture positivity and drug resistance in abdominal tuberculosis in Mumbai, India. Indian J Gastroenterol. 2014 Sep;33(5):414-9.

24 Mehta V, Desai D, Abraham P, Gupta T, Rodrigues C, Joshi A, et al. Do additional colonoscopic biopsies increase the yield of Mycobacterium tuberculosis culture in suspected ileo-colonic tuberculosis? Indian J Gastroenterol. 2018 May;37(3):226-30.

25 Leung VK, Law ST, Lam CW, Luk IS, Chau $\mathrm{TN}$, Loke TK, et al. Intestinal tuberculosis in a regional hospital in Hong Kong: a 10-year experience. Hong Kong Med J. 2006 Aug; 12(4):264-71

26 Makharia GK, Srivastava S, Das P, Goswami $\mathrm{P}$, Singh U, Tripathi $\mathrm{M}$, et al. Clinical, endoscopic, and histological differentiations between Crohn's disease and intestinal tuberculosis. Am J Gastroenterol. 2010 Mar;105(3): 642-51.

27 Ko JK, Lee HL, Kim JO, Song SY, Lee KN, Jun DW, et al. Visceral fat as a useful parameter in the differential diagnosis of Crohn's disease and intestinal tuberculosis. Intest Res. 2014 Jan;12(1):42-7.

28 Yadav DP, Madhusudhan KS, Kedia S, Sharma R, Pratap Mouli V, Bopanna S, et al. Development and validation of visceral fat quantification as a surrogate marker for differen- tiation of Crohn's disease and intestinal tuberculosis. J Gastroenterol Hepatol. 2017 Feb;32(2):420-6.

29 Hillemann D, Galle J, Vollmer E, Richter E. Real-time PCR assay for improved detection of Mycobacterium tuberculosis complex in paraffin-embedded tissues. Int J Tuberc Lung Dis. 2006 Mar;10(3):340-2.

30 Pulimood AB, Peter S, Rook GW, Donoghue HD. In situ PCR for Mycobacterium tuberculosis in endoscopic mucosal biopsy specimens of intestinal tuberculosis and Crohn disease. Am J Clin Pathol. 2008 Jun;129(6):846-51.

31 Gan HT, Chen YQ, Ouyang Q, Bu H, Yang $X Y$. Differentiation between intestinal tuberculosis and Crohn's disease in endoscopic biopsy specimens by polymerase chain reaction. Am J Gastroenterol. 2002 Jun;97(6): 1446-51.

32 Lodha R, Kabra SK. Newer diagnostic modalities for tuberculosis. Indian J Pediatr. 2004 Mar;71(3):221-7.

33 Blakemore R, Nabeta P, Davidow AL, Vadwai V, Tahirli R, Munsamy V, et al. A multisite assessment of the quantitative capabilities of the Xpert MTB/RIF assay. Am J Respir Crit Care Med. 2011 Nov;184(9):1076-84.

34 Pimkina E, Zablockis R, Nikolayevskyy V, Danila E, Davidaviciene E. The Xpert ${ }^{\circledR}$ MTB/ RIF assay in routine diagnosis of pulmonary tuberculosis: A multicentre study in Lithuania. Respir Med. 2015 Nov;109(11):1484-9.

35 Singh UB, Pandey P, Mehta G, Bhatnagar AK, Mohan A, Goyal V, et al. Genotypic, Phenotypic and Clinical Validation of GeneXpert in Extra-Pulmonary and Pulmonary Tuberculosis in India. PLoS One. 2016 Feb; 11(2):e0149258.

36 Kumar S, Bopanna S, Kedia S, Mouli P, Dhingra R, Padhan R, et al. Evaluation of Xpert MTB/RIF assay performance in the diagnosis of abdominal tuberculosis. Intest Res. 2017 Apr;15(2):187-94.

37 Kumar P, Pandya D, Singh N, Behera D, Aggarwal P, Singh S. Loop-mediated isothermal amplification assay for rapid and sensitive diagnosis of tuberculosis. J Infect. 2014 Dec;69(6):607-15.

38 Bojang AL, Mendy FS, Tientcheu LD, Otu J, Antonio M, Kampmann B, et al. Comparison of TB-LAMP, GeneXpert MTB/RIF and culture for diagnosis of pulmonary tuberculosis in The Gambia. J Infect. 2016 Mar;72(3):3327.

39 Zhang F, Xu C, Ning L, Hu F, Shan G, Chen $\mathrm{H}$, et al. Exploration of serum proteomic profiling and diagnostic model that differentiate Crohn's disease and intestinal tuberculosis. PLoS One. 2016 Dec;11(12):e0167109.

40 Rukmangadachar LA, Makharia GK, Mishra A, Das P, Hariprasad G, Srinivasan A, et al. Proteome analysis of the macroscopically affected colonic mucosa of Crohn's disease and intestinal tuberculosis. Sci Rep. 2016 Mar; 6(1):23162. 